\title{
How Physically Active Are People with Stroke in Physiotherapy Sessions Aimed at Improving Motor Function? A Systematic Review
}

\author{
Gurpreet Kaur, ${ }^{1}$ Coralie English, ${ }^{1,2}$ and Susan Hillier ${ }^{1,2}$ \\ ${ }^{1}$ School of Health Sciences, University of South Australia, P.O. Box 2471, Adelaide, SA 5001, Australia \\ ${ }^{2}$ International Centre for Allied Health Evidence, School of Health Sciences, University of South Australia, \\ P.O. Box 2471, Adelaide, SA 5001, Australia
}

Correspondence should be addressed to Coralie English, coralie.english@unisa.edu.au

Received 7 October 2011; Revised 19 December 2011; Accepted 12 January 2012

Academic Editor: Ching-yi Wu

Copyright (C) 2012 Gurpreet Kaur et al. This is an open access article distributed under the Creative Commons Attribution License, which permits unrestricted use, distribution, and reproduction in any medium, provided the original work is properly cited.

\begin{abstract}
Background. Targeted physical activity drives functional recovery after stroke. This review aimed to determine the amount of time stroke survivors spend physically active during physiotherapy sessions. Summary of Review. A systematic search was conducted to identify published studies that investigated the use of time by people with stroke during physiotherapy sessions. Seven studies were included; six observational and one randomised controlled trial. People with stroke were found to be physically active for an average of 60 percent of their physiotherapy session duration. The most common activities practiced in a physiotherapy session were walking, sitting, and standing with a mean (SD) practice time of 8.7 (4.3), 4.5 (4.0), and 8.3 (2.6) minutes, respectively. Conclusion. People with stroke were found to spend less than two-thirds of their physiotherapy sessions duration engaged in physical activity. In light of dosage studies, practice time may be insufficient to drive optimal motor recovery.
\end{abstract}

\section{Introduction}

People with stroke spend less than a quarter of their day engaged in physical activity in rehabilitation centres $[1,2]$. Studies conducted internationally over many years have shown that the time spent by people with stroke in therapy and in contact with therapists during the working day is very little [1-4]. Bernhardt et al. [1] found that people with stroke spent only 5.2 percent of the working day in contact with therapists in an acute stroke unit which equates to 0.5 hours of a nine-hour observation period. Similarly, Thompson and McKinstry [4] found that people with stroke in an inpatient rehabilitation unit spent only 1.2 hours of an 11-hour observation period in therapy. However, an observational study investigating the use of time in physiotherapy sessions specifically, reported that people with stroke spend between 21 percent and 30 percent of therapy sessions inactive [5]. Many studies have identified therapy sessions as being the most active part of the day and therefore provide the greatest opportunity to maximise physical activity levels. Therefore, this review was important to determine exactly how active people with stroke are in their physiotherapy sessions during stroke rehabilitation.

Targeted physical activity drives functional recovery after stroke. There is now strong evidence that more time spent in task-specific therapy after stroke improves functional outcomes $[6,7]$. Furthermore, high repetitions of a task-specific physical activity have been shown to facilitate positive neuroplasticity in stroke survivors [8-10].

In light of this knowledge, and the fact that clinical guidelines for stroke management include recommendations to maximise active therapy time [11], it is important to determine just how active stroke survivors are in therapy sessions aimed at improving motor function. Therefore, the aim of this review was to synthesise the current evidence about the total amount of time spent by people with stroke engaged in physical activity (total active time) and time spent engaged in different physical activity categories during physiotherapy sessions in stroke rehabilitation. 
TABLE 1: Search terms used to identify published studies which reported data on therapy content and duration.

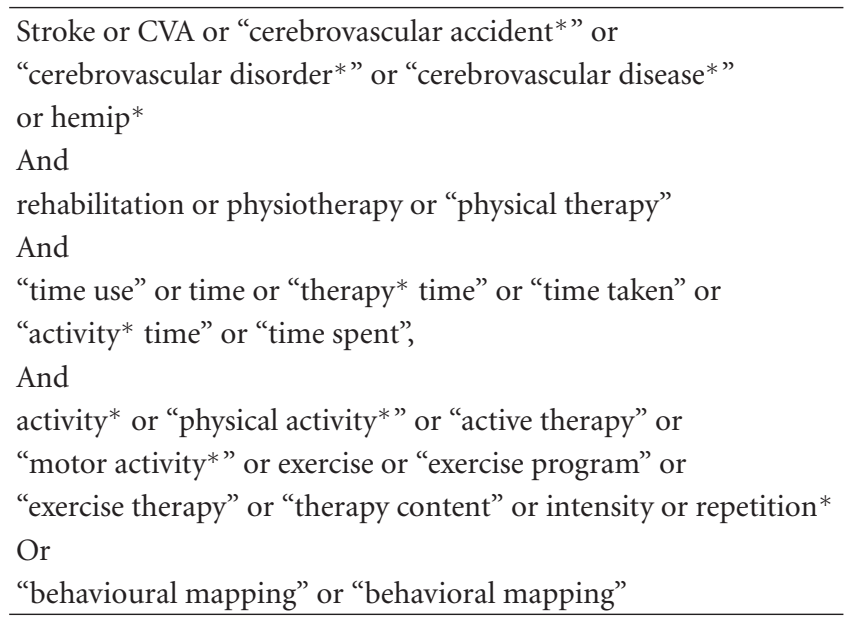

\section{Methods}

Published studies of all designs were identified by entering various search terms (Table 1) in the following electronic databases: CINAHL, AMED, MEDLINE, and EMBASE (Classic + Embase from 1947 to 2010, week 38). Preliminary searches were conducted in August 2010 to find the appropriate search terms. The final search was completed on the 11 th of December, 2011. The reference lists of studies which met the selection criteria were searched for potentially relevant publications. Searches were limited by years of publication (1990 to current), English language, and participants' age group (18 years and over). The authors of studies which reported both therapy content and therapy time but had insufficient data for the purposes of this systematic review were contacted for additional data.

Studies were included if (1) participants were adults receiving rehabilitation after stroke, (2) physiotherapy was provided in inpatient rehabilitation or an acute care hospital, and (3) data relating to both the therapy content (type of physical activity subcategories), and the amount of time spent in different physical activity categories during physiotherapy sessions was reported (i.e., studies reporting content of therapy only or total duration of therapy sessions only were not included).

Critical appraisal of the relevant articles was conducted using a modified evidence-based learning critical appraisal checklist [12]. This tool assesses the quality of observational studies in four categories: population, data collection, study design, and results. The factors which were most likely to contribute bias to the studies were chosen from the original critical appraisal tool. Two additional criteria were added: "concealment of study purpose to the participants" as this would reduce any bias arising from changes in usual practice and "involvement of an independent person observing the therapy sessions" which would reduce the risk of bias of overestimation of therapy duration. A copy of the critical appraisal tool as it was applied in this review appears in the Appendix. Two reviewers critically appraised the included studies independently. Justification of results from both reviewers was discussed and when disagreement occurred, a consensus was reached by discussion. Where further information was required to clarify criteria (e.g., whether the assessor was an independent person), authors were contacted directly. Where consensus could not be reached or criteria remained ambiguous, the opinion of a third reviewer was sought.

Data from the included studies were extracted including total therapy session duration, time spent in different physical activity subcategories, and total inactive time. The percentage of total active time was calculated using total time spent in physical activity subcategories in a therapy session. The total active time was averaged across studies, where data were sufficiently homogenous.

\section{Results}

The search yielded 2534 hits of which 61 studies were potentially relevant after reviewing titles and abstract (see Figure 1 for flow chart). After removing 13 duplicates, 48 relevant full-text articles were obtained and assessed against the selection criteria by one reviewer. A total of 28 studies were excluded because of their outpatient setting, and/or lack of data on therapy content or duration. Two reviewers assessed the full text of the remaining 20 studies and independently made a decision about studies to be included in the review. A third reviewer was available to adjudicate any disagreements, but this was not required. The reference lists of the included seven studies were scrutinized, but no further relevant publications were identified. An expert check was also conducted, but no further studies were identified.

All of the included studies were assessed as having a low risk of bias except Peurala et al. [14] which was assessed as having moderate to high risk of bias (Table 2). In Peurala et al. [14], there were no independent observers involved (the same therapists that provided the therapy also evaluated content and duration), the data collection methods were not adequately described, and the data collection instrument was not validated. Furthermore, it was unclear if the study purpose was concealed from the participants and therapists, thereby increasing the risk of overestimation of activity levels.

All the included studies were observational except Peurala et al. [14], which was an RCT. The majority of the included studies were conducted in Australia (Table 3). Five of the included studies were carried out in inpatient rehabilitation facilities and two in acute care hospitals (Table 3 ). The average time since stroke ranged from $5.6[15]$ to 161 [16] days (Table 3). Video recording, behavioural mapping, and contemporaneous recording by the researchers or therapists were the three main methods of observation used in the included studies. In the video recording method of observation, the entire physiotherapy session was video-recorded and later analysed to extract the data. In the behavioural mapping method of observation, the total observation period (for, e.g., from 9 am to $5 \mathrm{pm}$ ) is 


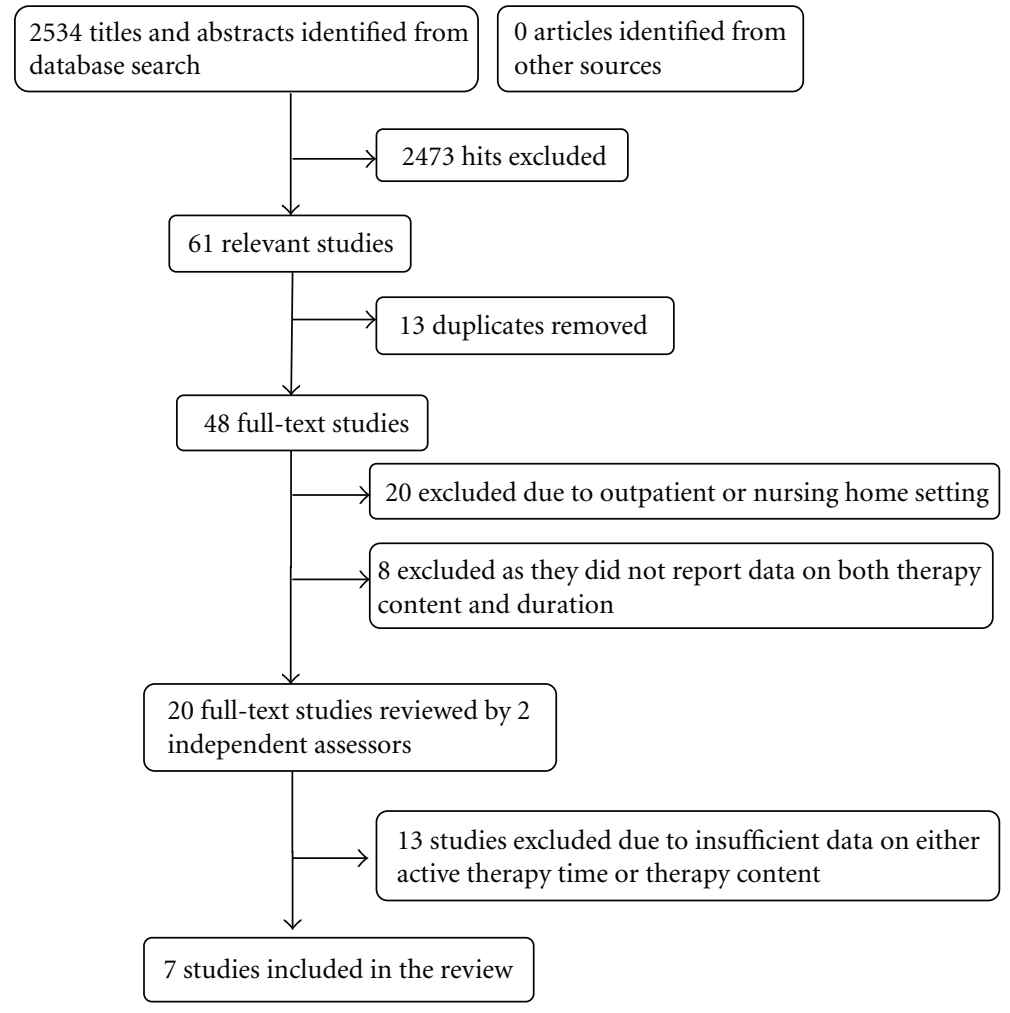

Figure 1: Flowchart of study selection (according to the Prisma statement [13]).

divided into 10-minute time slots. During every 10 minutetime slot, participants are observed for one minute, and their behaviour is recorded. The contemporaneous recording involves researchers or treating therapists recording the content and duration of the therapy sessions in a data collection instrument during or after the observed therapy session.

The mean total therapy session time ranged from 24 minutes to 64 minutes (mean of 49.5 minutes) except Lang et al. [17] (Table 3). Lang et al. [17] measured the number of repetitions performed by participants during a therapy session but they did not report total therapy time. The amount of active time within physiotherapy sessions ranged from 27 minutes to 39 minutes (mean of 32.8 minutes). The percentage of time spent active during a therapy session ranged from 42 percent to 71 percent (mean of 60 percent) (Table 3). The time spent by people with stroke engaged in various physical activity subcategories is outlined in Table 4 .

The descriptions of physical activity subcategories and inactivity across the studies varied, but could be collated into categories of walking, standing activities, cycling, transfers (including sit-to-stand), sitting activities, upper limb activities, bed mobility/activities in lying, and inactivity/rest time.

3.1. Walking Practice. The average amount of time spent in walking practice ranged from four to 14.7 minutes per therapy session (Table 4). Walking practice included early gait activities (walking on level surface, therapist assisted using a gait aid) and advanced gait activities (walking on uneven surface, stairs, obstacle courses, and treadmill) [1416]. In the study by Lang et al. [17], the average number of steps taken per therapy session was 395 , and the average number of times the participants got up to walk was nine per therapy session.

3.2. Sitting Activities. All the studies except Lang et al. [17] reported the amount of time spent in sitting activities specifically. Sitting activities included postural control exercises, reaching, and active lower limb exercises. The average amount of time spent in sitting practice ranged from 0.3 to 9.6 minutes.

3.3. Standing Activities. All the studies except Lang et al. [17] reported the amount of time spent in standing activities specifically. Exercises in standing included standing up, shifting weight from one leg to the other, and reaching in standing. The average amount of time spent in standing practice ranged from four to 11.6 minutes per therapy session.

3.4. Upper Limb Activities. All the included studies except De Wit et al. [18] reported the amount of time spent in upper limb activities during therapy sessions. Upper limb activities included active task practice using the paretic upper limb. The average amount of time spent in upper limb activities ranged from 0.9 to 7.9 minutes per therapy session. Lang et al. [17] reported an average of 86 repetitions of active upper limb activities performed per therapy session. 
TABLE 2: Critical appraisal of the included studies.

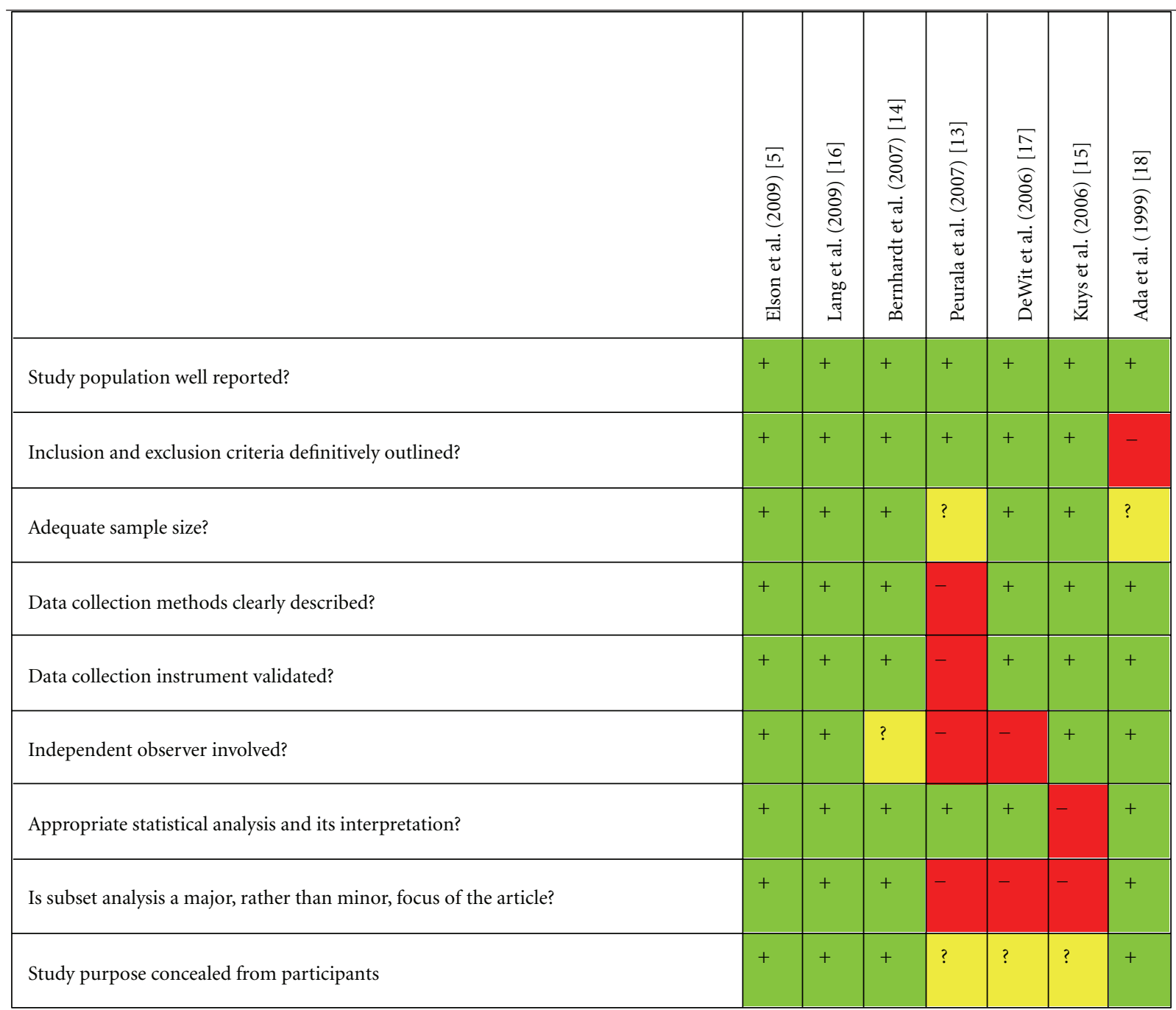

Key:

\begin{tabular}{llll}
\hline+ & Yes & No & $\square$ Unclear
\end{tabular}

3.5. Bed Mobility/Activities in Lying. Only three studies [5, $15,16]$ reported the average amount of time spent per therapy session performing bed mobility/activities in lying, this ranged from 0.3 to 5.2 minutes. Bed mobility/activities in lying included rolling, bridging, lying down from sitting, moving across the bed, sitting from lying, and isolated hip/knee control.

3.6. Transfers Including Sit-to-Stand Practice. Five of the included studies $[5,14,15,17,19]$ reported the amount of time/repetitions spent engaged in transfer exercises per therapy session. Transfers included sit-to-stand, moving from wheelchair to bed, and wheelchair to toilet. The average amount of time spent in transfer practice ranged from 1.8 to 3.7 minutes per therapy session. An average of 11 repetitions of transfers per session was reported by Lang et al. [17].

3.7. Other Therapeutic Activities. Only four of the included studies $[5,14,18,19]$ reported the time spent in other activities. Other therapeutic activities included cycling, activities in kneeling, passive movements, soft tissue techniques, tonus inhibition, positioning to stretch muscles, exercising the affected lower limb in any position, selective movements including coordination, strengthening exercises, and active relaxation. The average amount of time spent engaged in other activities ranged from 4.1 to 16 minutes. 


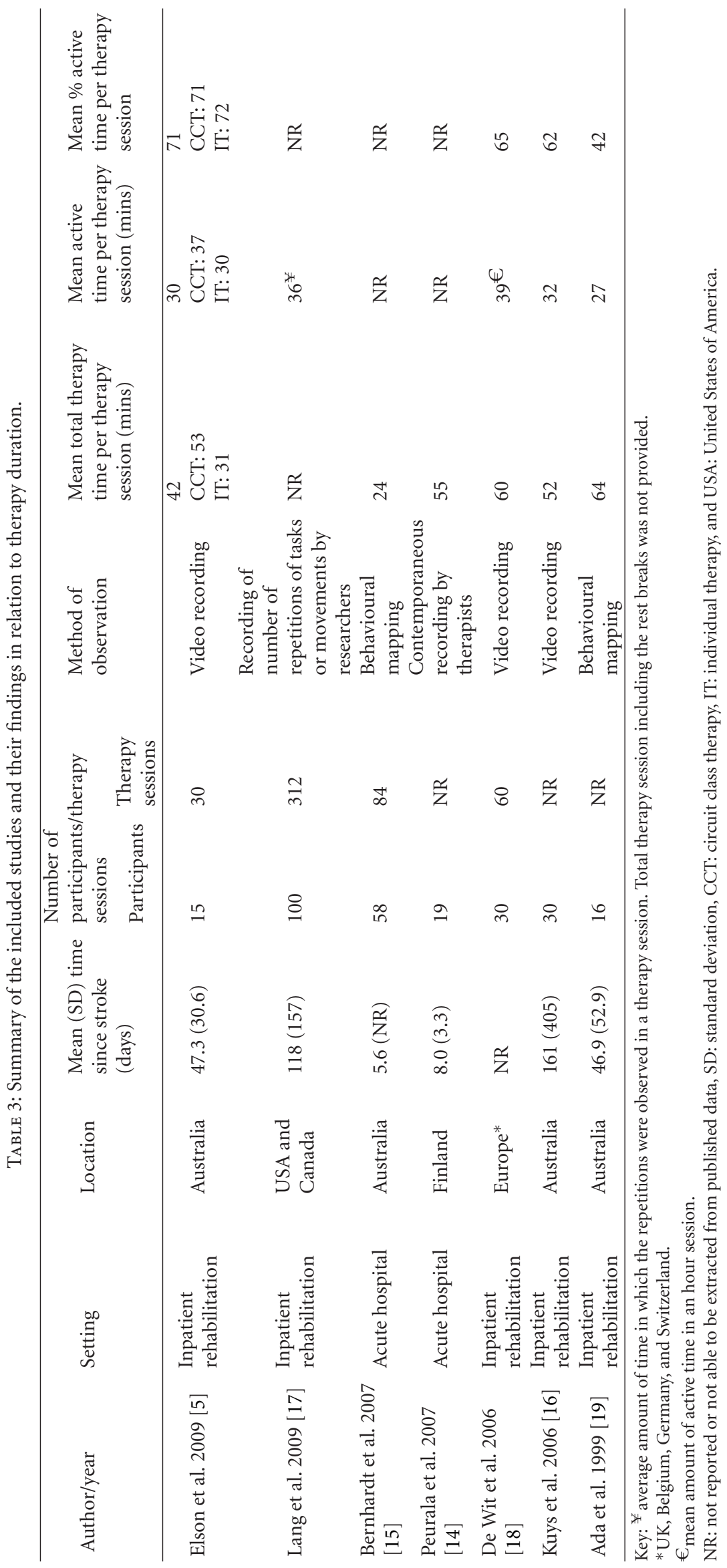


TABLE 4: The average amount of time spent by people with stroke engaged in different physical activity subcategories and time spent inactive in therapy sessions.

\begin{tabular}{|c|c|c|c|c|c|c|c|c|}
\hline Author/year & $\begin{array}{l}\text { Walking } \\
\text { practice }\end{array}$ & $\begin{array}{l}\text { Sitting } \\
\text { exercises }\end{array}$ & $\begin{array}{l}\text { Standing } \\
\text { exercises }\end{array}$ & $\begin{array}{c}\text { Upper } \\
\text { limb } \\
\text { activities }\end{array}$ & $\begin{array}{l}\text { Bed mobil- } \\
\text { ity/activities in } \\
\text { lying }\end{array}$ & $\begin{array}{c}\text { Transfers and } \\
\text { sit-to-stand } \\
\text { practice }\end{array}$ & $\begin{array}{l}\text { Other } \\
\text { therapeutic } \\
\text { activities } €\end{array}$ & $\begin{array}{c}\text { Inactive } \\
\text { time }\end{array}$ \\
\hline Elson et al. 2009 [5] & 10.8 & 0.8 & 8.5 & 0.9 & 0.3 & 2.7 & 5.0 & 12.7 \\
\hline Bernhardt et al. 2007 [15] & 4.7 & 5.7 & 4.0 & 2.8 & 2.8 & 1.8 & NR & NR \\
\hline Peurala et al. 2007 [14] & 14.7 & 9.6 & 10.4 & 1.2 & NR & 3.7 & 14.5 & NR \\
\hline De Wit et al. 2006 [18] & 6.4 & 8.4 & 7.6 & NR & NR & NR & 16.0 & NR \\
\hline Kuys et al. 2006 [16] & 11.8 & 2.1 & 11.6 & 2.9 & 5.2 & NR & NR & 12.4 \\
\hline Ada et al. 1999 [19] & 4.0 & 0.3 & 7.5 & 7.9 & NR & 3.2 & 4.1 & 37 \\
\hline
\end{tabular}

Key: NR: not reported.

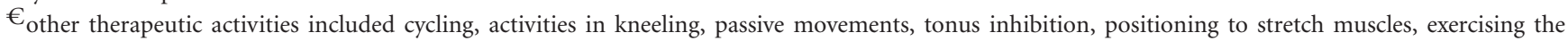
affected lower limb in any position, selective movements including coordination, strengthening exercises, and active relaxation.

Total active time after adding up the time spent in each activity subcategory might not be the same as Table 3 due to rounding, and/or inaccuracy of data reported in the included studies.

The data from Lang et al. [17] included the number of repetitions of various physical activity subcategories, and, therefore, was not included in this table.

3.8. Inactive/Rest Time. Only three studies $[5,16,19]$ investigated the amount of inactive time during therapy sessions. The inactive periods included time spent resting between activities, receiving instruction from or waiting for the therapist, and use of the nonparetic upper limb/lower limb. The average amount of inactive time ranged from 12.4 to 37 minutes per therapy session.

Figure 2 provides a visual representation of the average amount of time spent engaged in each category of activity in the context of total therapy time and enables a comparison with inactive or rest time.

\section{Discussion}

This systematic review found that people with stroke spent more than a third (40 percent) of their physiotherapy sessions inactive. In other words, 60 percent of the total therapy session duration was the averaged total active time. Physiotherapy techniques like passive movement, tonus inhibition, and stretching were included in "other therapeutic activities" within active time; therefore, this figure of 40 percent constitutes absolute rest or inactive time. Inconsistencies were found in how others have categorised these techniques as either passive or active, so the most conservative interpretation was chosen. The mean amount of active time in physiotherapy sessions was 32.8 minutes. The most common physical activities observed during physiotherapy sessions were walking, sitting, and standing practice. However, the actual amount of time spent by people with stroke engaged in these activities per therapy session was small (mean of 8.7 (SD 4.3) minutes walking practice, 4.5 (4.0) minutes sitting practice, and 8.3 (2.6) minutes standing practice).

Even less time was spent per therapy session on activities such as upper limb practice, transfers, and bed mobility. While we do not yet know what the optimal dose of therapy time and repetitions is to optimise functional recovery after stroke, neuroplasticity literature suggests that hundreds to thousands of repetitions of a task or movement are required to lead to lasting neural changes [20-23]. This raises the question as to whether the amount of task-specific practice currently provided during stroke rehabilitation is adequate to drive neuroplastic changes for optimal functional recovery.

Overall, the quality of the included studies was moderateto-high with the exception of Peurala et al. [14] in which the method of data collection was not clearly described or validated. The study relied on therapists recording of the content of physiotherapy sessions which may not be accurate. Therapists are known to systematically overestimate the time participants spend in therapy sessions [24]. We recently completed a study examining the accuracy of physiotherapists in estimating both therapy duration and the time spent in different categories of activity and inactivity in therapy sessions and found therapists systematically overestimated active time by 28 percent and systematically underestimated inactive time by 36 percent (unpublished data). This suggests any studies relying on therapist reports of therapy sessions are likely to overestimate active time. All other studies in the review used more objective measures of estimating active time in physiotherapy sessions. Two studies $[15,19]$ used behavioural mapping - a well established method by which participants are observed for one minute out of every 10 minutes per day and their activities are recorded. This method poses some limitations as the participants were observed for only one minute out of every 10 minutes during the total observation period. All other studies used some form of continuous monitoring of physiotherapy sessionseither by videoing sessions in their entirety and analysing the footage later $[5,16,18]$ or by counting repetitions of a particular task [17]. While these methods are likely to provide a more accurate picture of therapy time, there remains a risk of bias due to a possible increase in activity levels of participants because they are obviously being observed throughout their therapy session. These limitations in study design are most likely to lead to an inflation of active time in physiotherapy sessions. 


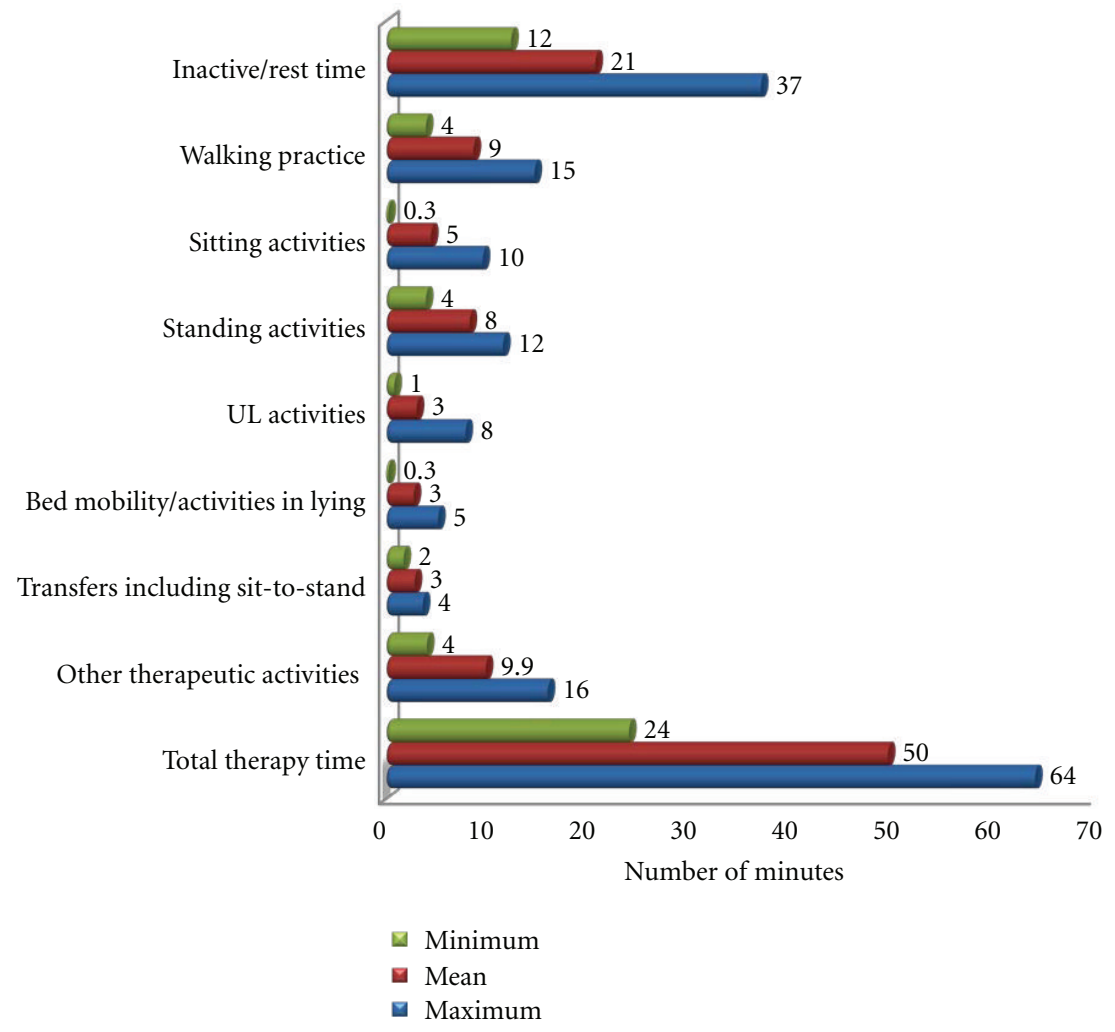

Figure 2: Mean amount of time spent in different physical activity subcategories, and the time spent inactive in relation to the mean total therapy time. The minimum and maximum time spent in different physical activities may not be identical to the data in Table 4 due to rounding.

Two of the included studies were conducted in acute settings and five in inpatient settings. The studies from outpatients and community settings were excluded because frequency and occasions of service delivery would be even more varied and therefore skew the results. Therefore, the findings of this review can only be generalised to services where the person is an inpatient. Even then there are some possible minor differences between acute and subacute settings in terms of length of therapy session provided. For this reason, we have concentrated on the percentage times spent active so that results can be more meaningfully interpreted.

The low level of physical activity within physiotherapy sessions is of concern, considering that clinical guidelines suggest that rehabilitation after stroke should be structured to provide practice time as much as possible, and that at least one hour per day should be spent in active task practice [11]. This review suggests that stroke survivors are doing only around 33 minutes of active task practice in each therapy session which falls well short of the recommended target if it is the only session provided during the day.

The included studies did not report therapy session frequency per day. Therefore, only findings for percentage of total active time per therapy session were summarised. It is possible, particularly in the acute setting, that participants received multiple therapy sessions per day. Further studies are needed to report total active time in therapy sessions for the entire day in line with current stroke guidelines.
Time spent physically active could be increased by increasing the total time spent by people with stroke in therapy sessions. Group circuit class therapy sessions are an alternative to individual physiotherapy sessions. They are longer in duration and therefore have the potential to increase active practice time. Elson et al. [5] reported that the time spent physically active by people with stroke was higher during circuit class therapy sessions due to their longer therapy duration. However, in percentage terms, the time spent by stroke participants engaged in physical activity during circuit class therapy sessions was similar to that of the individual therapy sessions [5]. Therefore, the effectiveness of circuit class therapy sessions in increasing physical activity levels of people with stroke requires further research.

There is a lack of evidence on the optimal dose and timing of therapy (block activity or frequent short bursts) required to enhance functional recovery after stroke. The optimal ways to schedule and deliver therapy is still unknown. However, therapists should aim to maximise the time people with stroke spend physically active in therapy sessions. Further research should focus on investigating the optimum timing and scheduling of therapy sessions.

4.1. Limitations. Only one person completed the first review of titles and abstracts which may have resulted in some potential papers being missed. Data on therapy content and 
duration could not be directly extracted from some studies [19] and required a process of extrapolation which may have introduced errors. The degree to which the data presented in the included studies is reflective of usual practice is not known. Finally, only data for physiotherapy sessions were located. Studies investigating physical activity time in other rehabilitation sessions (such as occupational therapy) should be conducted to give a more comprehensive picture of the person's overall therapy experience.

\section{Conclusion}

The findings of this systematic review suggest that people with stroke are engaged in physical activity for less than twothirds of the total physiotherapy session duration. The time spent by people with stroke engaged in the most commonly observed physical activity categories (walking, sitting, and standing) may be lower than what is recommended, and that is reported to be required to drive positive neuroplastic changes and optimise functional recovery. Therefore, therapists should be aware of the likelihood that people with stroke might not be receiving enough practice time. Researchers need to investigate the optimal therapy intensity and timing of therapy to drive positive neuroplasticity and functional recovery after stroke.

\section{Appendix}

\section{Modified Evidence-Based Learning Critical Appraisal Checklist}

(i) Is the study population well reported? (i.e., acuity, and severity of stroke)

(ii) Are the inclusion and exclusion criteria definitively outlined?

(iii) Is the sample large enough for sufficiently precise estimates?

(iv) Are data collection methods clearly described?

(v) Is the data collection instrument validated?

(vi) Were those involved in data collection not involved in delivering a service to the target population?

(vii) Is the statistical analysis and its interpretation appropriate?

(viii) Is the subset analysis a major, rather than a minor, focus of the paper?

(ix) Was the study purpose concealed from the participants?

\section{References}

[1] J. Bernhardt, H. Dewey, A. Thrift, and G. Donnan, "Inactive and alone: physical activity within the first 14 days of acute stroke unit care," Stroke, vol. 35, no. 4, pp. 1005-1009, 2004.

[2] L. De Wit, K. Putman, E. Dejaeger et al., "Use of time by stroke patients: a comparison of four European rehabilitation centers," Stroke, vol. 36, no. 9, pp. 1977-1983, 2005.
[3] W. De Weerdt, B. Selz, G. Nuyens et al., "Time use of stroke patients in an intensive rehabilitation unit: a comparison between a Belgian and a Swiss setting," Disability and Rehabilitation, vol. 22, no. 4, pp. 181-186, 2000.

[4] R. Thompson and W. McKinstry, "How do rehabilitation patients spend their days? An investigation into the effect of a group therapy programme on time use," New Zealand Journal of Physiotherapy, vol. 37, no. 3, pp. 122-126, 2009.

[5] T. Elson, C. English, and S. Hillier, "How much physical activity do people recovering from stroke do during physiotherapy sessions?" International Journal of Therapy and Rehabilitation, vol. 16, no. 2, pp. 78-84, 2009.

[6] G. Kwakkel, R. V. Peppen, R. C. Wagenaar et al., "Effects of augmented exercise therapy time after stroke: a meta-analysis," Stroke, vol. 35, no. 11, pp. 2529-2536, 2004.

[7] R. P. S. Van Peppen, G. Kwakkel, S. Wood-Dauphinee, H. J. M. Hendriks, P. J. Van der Wees, and J. Dekker, "The impact of physical therapy on functional outcomes after stroke: what's the evidence?" Clinical Rehabilitation, vol. 18, no. 8, pp. 833862, 2004.

[8] J. Liepert, W. H. Miltner, H. Bauder et al., "Motor cortex plasticity during constraint,induced movement therapy in stroke patients," Neuroscience Letters, vol. 250, no. 1, pp. 5-8, 1998.

[9] J. Liepert, S. Graef, I. Uhde, O. Leidner, and C. Weiller, "Training-induced changes of motor cortex representations in stroke patients," Acta Neurologica Scandinavica, vol. 101, no. 5, pp. 321-326, 2000.

[10] J. R. Carey, T. J. Kimberley, S. M. Lewis et al., "Analysis of fMRI and finger tracking training in subjects with chronic stroke," Brain, vol. 125, no. 4, pp. 773-788, 2002.

[11] National Stroke Foundation, "Clinical Guidelines for Stroke Management," 2010, http://www.strokefoundation.com.au/ clinical-guidelines.

[12] L. Glynn, "EBLIP Critical Appraisal Checklist," Memorial University of Newfoundland, Canada, 2006, http://www.nihs.ie/ pdf/EBL Critical Appraisal Checklist.pdf.

[13] D. Moher, A. Liberati, J. Tetzlaff, D. G. Altman, and The PRISMA Group, "Preferred reporting items for systematic reviews and meta-analyses: the PRISMA statement," PLoS Medicine, vol. 6, no. 7, Article ID e1000097, 2009.

[14] S. H. Peurala, O. Airaksinen, P. Jäkälä, I. M. Tarkka, and J. Sivenius, "Effects of intensive gait-oriented physiotherapy during early acute phase of stroke," Journal of Rehabilitation Research and Development, vol. 44, no. 5, pp. 637-648, 2007.

[15] J. Bernhardt, J. Chan, I. Nicola, and J. M. Collier, "Little therapy, little physical activity: rehabilitation within the first 14 days of organized stroke unit care," Journal of Rehabilitation Medicine, vol. 39, no. 1, pp. 43-48, 2007.

[16] S. Kuys, S. Brauer, and L. Ada, "Routine physiotherapy does not induce a cardiorespiratory training effect poststroke, regardless of walking ability," Physiotherapy Research International, vol. 11, no. 4, pp. 219-227, 2006.

[17] C. E. Lang, J. R. MacDonald, D. S. Reisman et al., "Observation of amounts of movement practice provided during stroke rehabilitation," Archives of Physical Medicine and Rehabilitation, vol. 90, no. 10, pp. 1692-1698, 2009.

[18] L. De Wit, K. Putman, N. Lincoln et al., "Stroke rehabilitation in Europe: what do physiotherapists and occupational therapists actually do?" Journal of The American Heart Association, vol. 37, no. 6, pp. 1483-1489, 2006.

[19] L. Ada, F. Mackey, R. Heard, and R. Adams, "Stroke rehabilitation: does the therapy area provide a physical challenge?" 
Australian Journal of Physiotherapy, vol. 45, no. 1, pp. 33-38, 1999.

[20] L. A. Boyd, E. D. Vidoni, and B. D. Wessel, "Motor learning after stroke: is skill acquisition a prerequisite for contralesional neuroplastic change?" Neuroscience Letters, vol. 482, no. 1, pp. 21-25, 2010.

[21] J. A. Kleim and T. A. Jones, "Principles of experiencedependent neural plasticity: implications for rehabilitation after brain damage," Journal of Speech, Language, and Hearing Research, vol. 51, no. 1, pp. S225-S239, 2008.

[22] C. Chau, H. Barbeau, and S. Rossignol, "Early locomotor training with clonidine in spinal cats," Journal of Neurophysiology, vol. 79, no. 1, pp. 392-409, 1998.

[23] J. Cha, C. Heng, D. J. Reinkensmeyer, R. R. Roy, V. R. Edgerton, and R. D. De Leon, "Locomotor ability in spinal rats is dependent on the amount of activity imposed on the hindlimbs during treadmill training," Journal of Neurotrauma, vol. 24, no. 6, pp. 1000-1012, 2007.

[24] P. Bagley, M. Hudson, J. Green, A. Forster, and J. Young, "Do physiotherapy staff record treatment time accurately? An observational study," Clinical Rehabilitation, vol. 23, no. 9, pp. 841-845, 2009. 


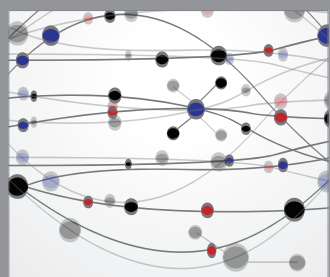

The Scientific World Journal
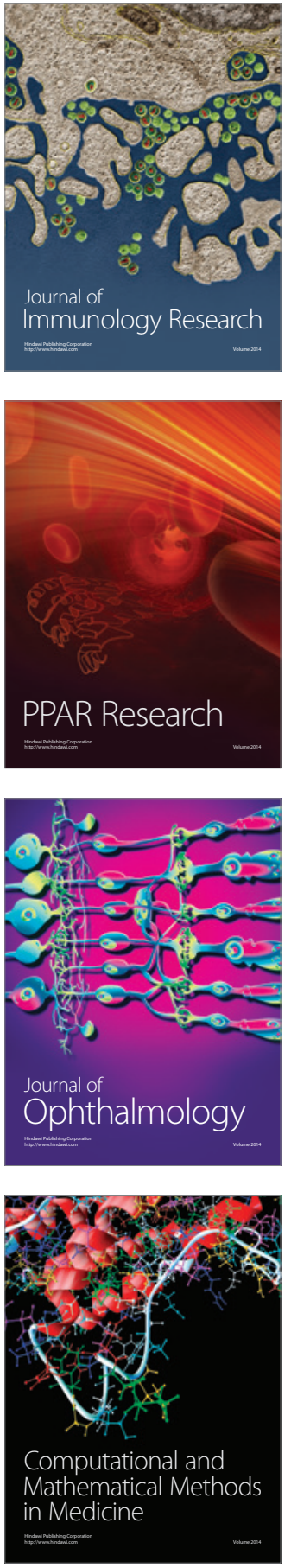

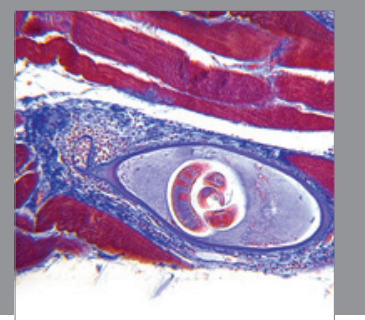

Gastroenterology

Research and Practice
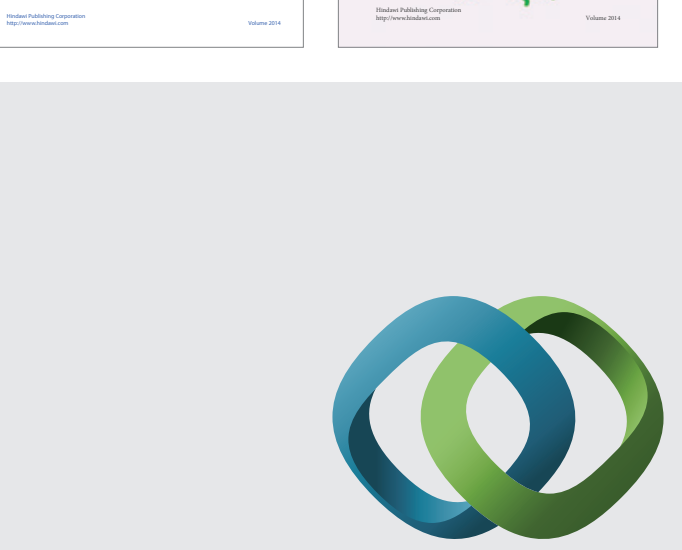

\section{Hindawi}

Submit your manuscripts at

http://www.hindawi.com
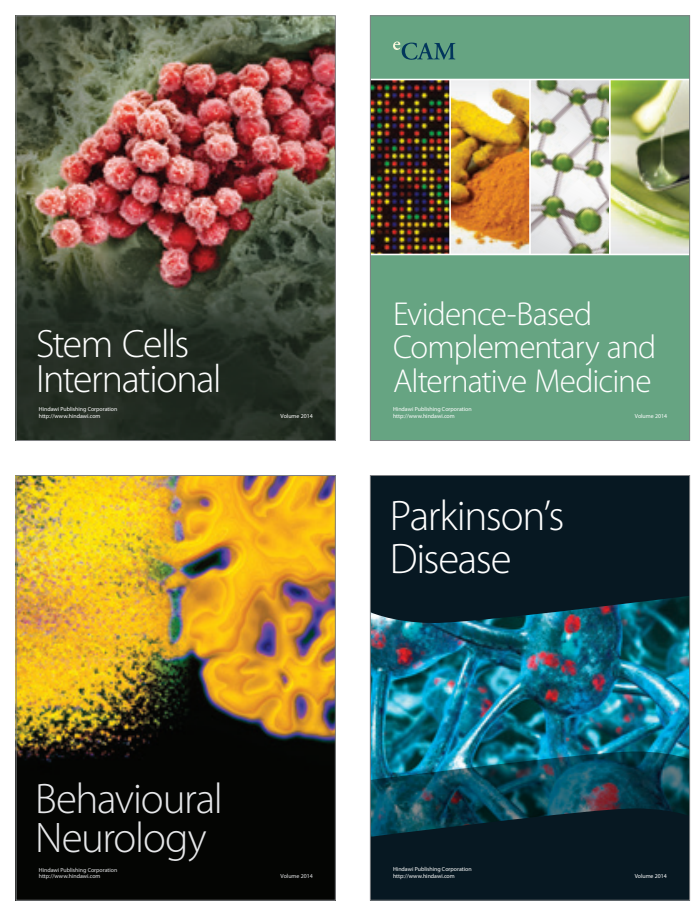

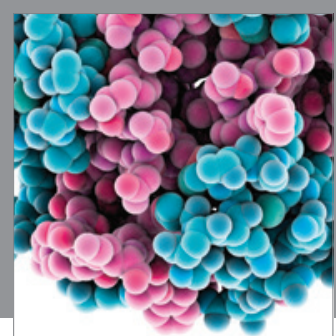

Journal of
Diabetes Research

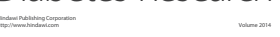

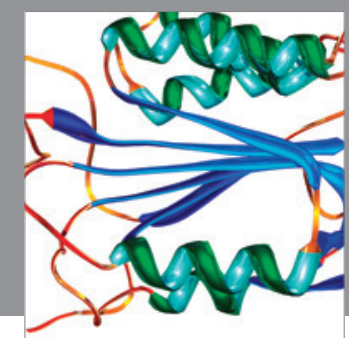

Disease Markers
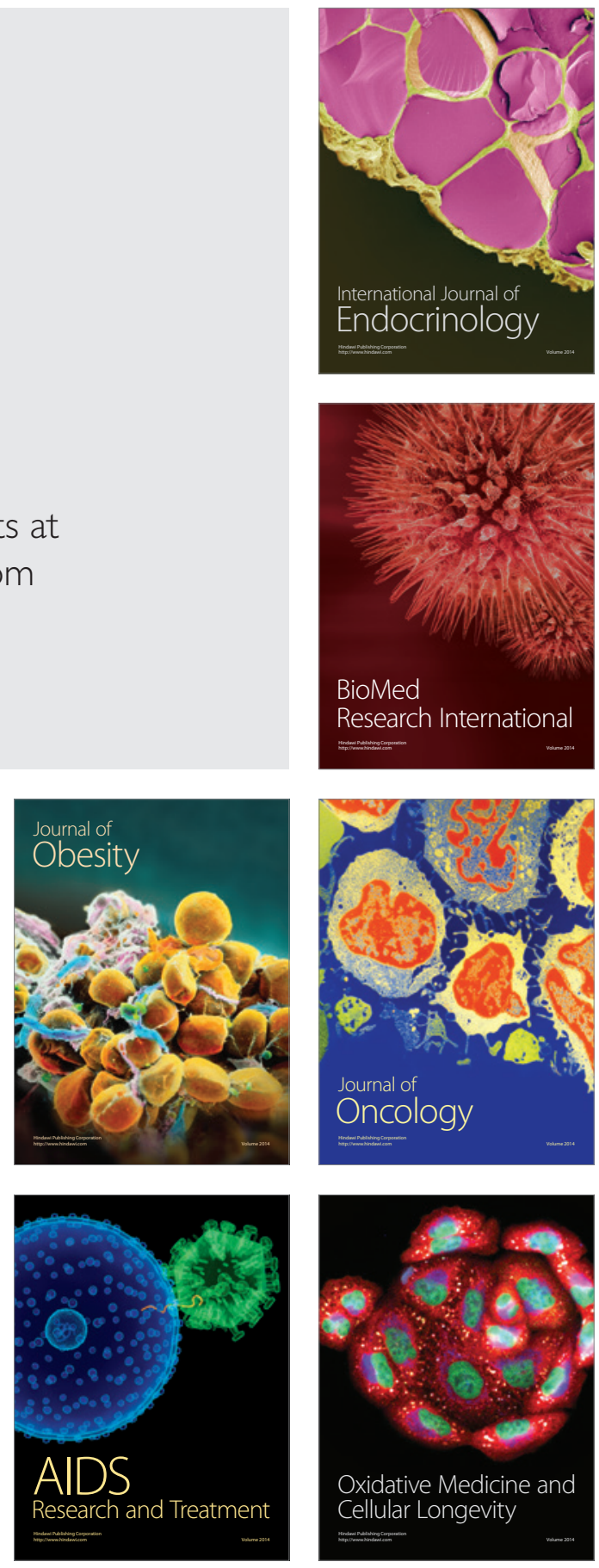